\title{
A NOTE ON WALLMAN COMPACTIFICATIONS
}

\author{
ASHA RANI SINGAL AND SUNDER LAL ${ }^{1}$
}

\begin{abstract}
T_{3}$ and $T_{3 \frac{1}{2}}$ spaces are characterized in terms of a Wallman compactification of a $T_{1}$ space $X$.
\end{abstract}

For a $T_{1}$ space $(X, \mathcal{T})$ consider the Wallman compactification $\left(\chi,\left(X^{*}, \mathcal{W}\right)\right)[3]$ consisting of the set $X^{*}$ of all ultraclosed filters on $(X, \mathcal{T})$, the topology $\mathscr{W}$ on $X^{*}$ generated by $\left\{U^{*}: U \in \mathcal{T}\right\}$ where $U^{*}=\left\{\mathcal{F} \in X^{*}: U \in \mathcal{F}\right\}$, and the dense embedding $\chi: X \rightarrow X^{*}$ defined by setting $\chi(x)=\delta(x)=\{A \subset X: x \in A\}$. A wellknown result about $\left(X^{*}, \mho\right)$ is that $(X, \mathcal{T})$ is $T_{4}$ iff $\left(X^{*}, \mho\right)$ is $T_{2}$. Here we characterize $T_{3}$ and $T_{3 \frac{1}{2}}$ spaces in a similar manner.

We define a space $(Y, Q)$ to be $T_{2}$ relative to $X$ for a subset $X$ of $Y$ if for each $x \in X$ and for each $y \in Y$ with $x \neq y$ there exist disjoint $\mathscr{Q}$-open sets $U$ and $V$ such that $x \in U$ and $y \in V .(Y, Q)$ is called completely $T_{2}$ relative to $X$ if for $x \in X$ and $y \in Y$ with $x \neq y$, there exists a continuous real-valued function for $Y$ with $f(x) \neq f(y)$.

THEOREM 1. $X$ is $T_{3 \frac{1}{2}}$ iff $X^{*}$ is completely $T_{2}$ relative to $\chi(X)$.

Proof. If $X^{*}$ is completely $T_{2}$ relative to $\chi(X)$, then $X$ is completely $T_{2}$. Let $F$ be a closed subset of $X$ and let $x \notin F$. Since $\chi$ is an embedding, $\chi(x) \notin \mathcal{Q}-\operatorname{cl} \chi(F)$. As $X^{*}$ is completely $T_{2}$ relative to $\chi(X)$, for each $y \in \mathcal{W}-\mathrm{cl} \chi(F)$, there exist disjoint cozero sets $U_{y}$ and $V_{y}$ in $X^{*}$ such that $y \in U_{y}, \chi(x) \in V_{y}$. Further $W-c l \chi(F)$, being a closed subset of $X^{*}$, is compact. Let $\left\{U_{y_{1}}, U_{y_{2}}, \ldots, U_{y_{n}}\right\}$ be a finite subcover of $\left\{U_{y}: y \in \mathcal{Q}-\mathrm{cl} \chi(F)\right\}$. Then $\cup_{i=1}^{n} U_{y_{i}}$ and $\cap_{i=1}^{n} V_{y_{i}}$ are disjoint cozero sets containing $\chi(F)$ and $\chi(x)$. Thus $x$ and $F$ are contained in disjoint cozero subsets of $X$ and, hence, $X$ is completely regular.

Conversely, let $X$ be $T_{3 \frac{1}{2}}$ and let $\mathscr{F} \in X^{*}$ and $\chi(x) \in \chi(X)$ be such that $\chi(x) \neq \mathscr{F}$. Since $\mathscr{F}$ is a closed ultrafilter, we have a closed subset $F \in \mathscr{F}$ such that $x \notin F$. Let a continuous $g: X \rightarrow[0,1]$ separate $x$ and $F$. If $g^{*}: X^{*} \rightarrow[0,1]$ is the continuous extension of $g$, then $g^{*}$ separates $\mathscr{F}$ and $\chi(x)$.

Using similar arguments one can prove

THEOREM 2. $X$ is $T_{3}$ iff $X^{*}$ is $T_{2}$ relative to $\chi(X)$.

Received by the editors September 5, 1980. Presented at the 46th annual conference of the Indian Mathematical Society held at Bangalore from December 27-29, 1980.

AMS (MOS) subject classifications (1970). Primary 54D35, 54D10, 54D15.

Key words and phrases. Wallman compactification, relatively $T_{2}$, relatively completely $T_{2}$, cozero sets.

${ }^{1}$ Research partially supported by grant no. CST/4657/79-MU(100)/78 from SCST Lucknow. 
In terms of the new definitions here the above result about $T_{4}$ spaces can be put down as

THEOREM 3. $X$ is $T_{4}$ iff $X^{*}$ is $T_{2}$ relative to $X^{*}$.

\section{REFERENCES}

1. L. Gillman and M. Jerison, Rings of continuous functions, Van Nostrand Reinhold, New York, 1960. 2. Darrel W. Hajek, $A$ characterization of $T_{3}$ spaces, Indiana Univ. Math. J. 23 (1973), 23-25.

3. W. J. Thron, Topological structures, Holt, Rinehart and Winston, New York, 1966.

Institute of AdVANCed Studies, Meerut University, Meerut-250001, INDIA 